In connection with the active development of information systems in transport, it becomes necessary to integrate vehicles, infrastructure and humans into a single information network. The V2I system of information analysis for monitoring and controlling vehicles in operating conditions is an organic combination of information and analytical components. The latter includes the analysis of information regarding changes in operating conditions. The article presents a study that has improved the processes of managing the operating conditions of vehicles in the V2I communication system by using simulation modelling. A simulation model for choosing the optimal operating conditions for vehicles is described. The model takes into account road, climatic, and transport conditions and culture of vehicle operation, as well as the peculiarities of public transport movement in a transport hub. The objective function with the appropriate restrictions and the problem of traffic optimization in the investigated transport hub were established. Diagrams of the processes of the simulation model were constructed for various input parameters, including the optimal ones, with the creation of corresponding agents and their populations. Models of public transport delays at stops using a triangular distribution were developed, and the corresponding hypotheses were confirmed by Pearson's test $\left(\chi^{2}\right)$. The developed models can be used in the process of rebuilding a transport hub, as well as for modeling traffic when the operating conditions of vehicles change and for predicting such changes. The simulation results can be used in the creation and design of intelligent transport systems

Keywords: simulation modelling, vehicle, transport hub, operating conditions, public transport, information system, intelligent transport system

Received date 18.09.2020

Accepted date 21.10.2020

Published date 30.10 .2020
UDC $656.1+004.94$

DOI: $10.15587 / 1729-4061.2020 .215006$

\section{DEVELOPMENT OF THE ANALYTICAL SYSTEM FOR VEHICLE OPERATING CONDITIONS MANAGEMENT IN THE V2I INFORMATION COMPLEX USING SIMULATION MODELING}

M. Volodarets

$\mathrm{PhD}$

Department of Electric Power Complexes and Systems*

I. Gritsuk

Doctor of Technical Sciences, Professor**

Y. Ukrainskyi

Senior Lecturer

Department of Automobile Transport*

E-mail: e.a.ukrainskyi@gmail.com

V. She in

$\mathrm{PhD}$

Department of Technology of Machinery Manufacturing and Machine Maintenance $* \star \star$

O. Stepanov

Doctor of Technical Sciences, Associate Professor Department of Traffic Management and Road Safety***

I. Khudiakov Senior Lecturer** M. A h i e i e v

$\mathrm{PhD}$, Associate Professor**

V. V y c h uzh a n in

Doctor of Technical Sciences, Professor Department of Information Technologies

Odessa National Polytechnic University

Shevchenka ave., 1, Odessa, Ukraine, 65044

O. S m y r n ov

Doctor of Technical Sciences, Associate Professor Department of Vehicle Electronics $* * *$

O. Saraiev

Doctor of Technical Sciences, Professor Department of Automobiles named after A. B. Hredeskul $* * *$ E-mail: sarayev9@gmail.com

*Pryazovskyi State Technical University Universytetska str., 2, Mariupol, Ukraine, 87555 **Department of Vessel's Power Plants Operation

Kherson State Maritime Academy

Ushakova ave., 20, Kherson, Ukraine, 73000 $\star * *$ Kharkiv National Automobile and Highway University Yaroslava Mudroho str., 25, Kharkiv, Ukraine, 61002

Copyright (C) 2020, M. Volodarets, I. Gritsuk, Y. Ukrainskyi, V. Shein, O. Stepanov, I. Khudiakov, M. Ahieiev, V. Vychuzhanin, O. Smyrnov, O. Saraiev This is an open access article under the CC BY license (http://creativecommons.org/licenses/by/4.0)
1. Introduction

The information analysis system of vehicle-to-infrastructure (V2I) communication to manage and control vehicles [1] in operating conditions is an effective combination of data and analytical components. The information part of the system consists in a connection of the vehicle's onboard control components and information facilities with 
the infrastructure's elements and systems of receiving and transmitting information, analysis, and control [1-10]. The analytical part includes software, logistical, and hardware components of the vehicle's board and infrastructure [1-4]. An obligatory element in this case is the place of the operator of the V2I communication network in the information analysis system for monitoring and controlling vehicles [1, 4, 5, 9] under the operating conditions. The components of the system as well as its general structure, operation and results of its information processing were described in detail in [1-10].

The operating conditions include climatic, road, and transport conditions and culture of vehicle operation [1].

The information part of the V2I system provides it with information from the vehicle and the infrastructure [1-10]. The analytical part of the V2I network processes information and controls the vehicle under operating conditions. One of the components of the analytical part is the analysis of information in terms of changes in the operating conditions, that is, in climatic, road, and transport conditions [1,11]. To ensure the systemic interaction of operating conditions and the vehicle in the described V2I system, the authors of the article propose a method for adapting computational procedures for information processing and traffic management using simulation modelling. It is best suited for research and management of road and transport operating conditions. It can also be used to research and control vehicles when the atmospheric and climatic conditions of operation and the culture of operation change.

Let us specify the features of the processes of research and management of the operating conditions of vehicles in the V2I information analysis system using simulation modelling.

The transport network is a complex system characterized by stochasticity, namely: a random value of transport demand, atmospheric and climatic factors, changes in the characteristics of the road network, as well as emergency situations and wear of the road surface $[1,12]$. Therefore, the most adequate means of describing and predicting the behaviour of the specified object is modelling, the essence of which is to replace the real control object with its simulation. Any object that reproduces the properties of a real system with sufficient accuracy for the user's purposes can act as a model. In recent years, information systems in transport have been actively developed. In this regard, it becomes necessary to integrate vehicles, infrastructure and humans into a single information system. One of the steps to solve this problem is to simulate the operating conditions of vehicles and their optimization. This will improve the control over vehicle operating conditions in the V2I communication system. This is especially useful in the context of the active development of intelligent transport systems.

\section{Literature review and problem statement}

Technical services for the operation of vehicles solve many problems in the process of improving the methods of operational management of vehicle performance. Most of the tasks have an information component of the assessment, such as to analyse road conditions of the vehicle operation; to predict possible emergency situations and transport conditions; to forecast atmospheric and climatic conditions, etc. [1,13-19]. The tasks listed and similar to them are still mainly solved by outdated methods that no longer provide the required quality, rational approach and efficiency [20-22]. Evaluation of operating conditions and analysis of road plans and profiles are usually performed manually; updates of maps and diagrams are extremely rare; data on the state of most objects are not systematized and thus difficult to access. This situation complicates the task of managing the classification of vehicle operating conditions in the information conditions of an ITS.

The process under consideration is complex; therefore, simulation modelling can be effective. Its main advantage is that, in contrast to analytical modelling, simulation modelling helps to repeatedly reproduce the studied complex system and determine its optimal performance.

Much research has been done in the field of transport modelling. Thus, in [1] an information system of interaction between the vehicle and the infrastructure was considered, but not enough attention was paid to modelling the transport and road conditions of the vehicle operation. In [2] a system of training for and safety of the vehicle in the conditions of intelligent transport systems was considered, but little attention was given to the influence of the operating conditions of the vehicle on its operation.

In [7] the general concept of developing intelligent transport systems was described but with little regard to the simulation modelling of the vehicle's operation under operating conditions. In [9] attention was paid only to the cyberprotection of vehicles in the context of intelligent transport systems, but the operating conditions of vehicles were poorly covered. Study [13] considered analytical models of urban transport, but it did not take into account the variety of operating conditions of the vehicles. In [19] methods for assessing the index of intelligent vehicles were considered, but without due attention to the influence of transport, road and atmospheric and climatic operating conditions of vehicles on their behaviour. In [23] modelling of road traffic of vehicles in the conditions of their operation was considered, but not enough attention was paid to the influence of the composition of vehicles on the adequacy of the traffic models. In [24] the issues of modelling the movement of intelligent vehicles were considered, but the influence of the culture of operating vehicles on their movement was not taken into account. In [25] the features of dynamic modelling and assessment of the state of an intelligent vehicle with several wheels and an engine were analysed, but the influence of climatic operating conditions and operating culture on the operation and behaviour of the vehicle was overlooked.

Therefore, it seems rational to conduct a study devoted to determining the mechanism for constructing a simulation model for choosing the optimal operating conditions for vehicles, taking into account the peculiarities of public transport. This will make it possible to use it for solving transport problems. In particular, the problem of optimizing the movement of vehicles can be solved. Moreover, it will be possible to predict the fuel consumption of vehicles with changing operating conditions.

\section{Research goals and objectives}

The aim of the work is to study the possibility of improving the processes of managing the operating conditions of vehicles in the information analysis system of the V2I communication network by using simulation modelling.

To achieve this aim, it is necessary to solve the following tasks: 
- to build a dynamic model for determining the optimal operating conditions for vehicles in a transport hub;

- to analyse the operating conditions of vehicles in the hub for which simulation modelling will be performed; and

- to build a simulation model for choosing the optimal operating conditions for vehicles for a section of the transport network.

\section{Construction of a dynamic model for determining the optimal operating conditions for vehicles in a transport hub}

A mathematical model for determining the optimal traffic parameters in a transport hub can be described as a correspondence between the elements of a set of inputs of the system $X$ of 'possible values' $x$ and a set $T$ of 'time points' $t$, that is, in the form of the following mapping of $T \rightarrow X$ : $x(t) \in X^{T}, t \in T$.

Considering the output $y(t)$ of the system as its reaction to inputs $x(t)$, we can represent the model as a set of two processes: $X^{T}=\{x(t)\}$ and $Y^{T}=\{y(t)\}, t \in T$ (Fig. 1). delay time of public transport at stops, $\operatorname{Cr}(t)$ is the state of the roadway, $T C_{i}(t)$ is the technical condition of the vehicles, $\operatorname{Pr}(t)$ is the road parameters, $C D(t)$ is the culture of vehicle operation, $C C(t)$ is the atmospheric and climatic conditions, $P I(t)$ is the infrastructure parameters, and $P S(t)$ is the parameters of the regulation and control system.

The set of output parameters of the model is represented by the following parameters: $p 1(t), p 2(t), p i(t)$, which are optimized parameters of vehicle operation.

The model can be used to develop a smart city transportation system to integrate the software into the infrastructure of the transport network.

\section{Analysis of the researched transport hub and the} operating conditions of its vehicles

Study [23] shows the cyphergram of the traffic flows in the transport hub (coordinates by Google Maps: 47 $57^{\prime} 31.6^{\prime \prime} \mathrm{N}$ $\left.37^{\circ} 48^{\prime} 22.6^{\prime \prime} \mathrm{E}\right)$. There, the traffic intensities were reduced. Based on the cyphergram, a graphical model of the movement of vehicles in the considered transport hub was built in the form of a graph of states with the probabilities of transitions from one state to another.

However, for modelling the movement of vehicles in the considered hub, as close as possible to the real one, it is necessary to select from the total number of public transport those vehicles that move within a given section with stops. Moreover, the article considers optimization of road and transport operating conditions as the most significant strategy. Other operating conditions are assumed constant.

Fig. 2 shows the transformed cyphergram for vehicles moving through the hub without stopping.

Fig. 3 shows a model of the movement of public transport with stops within the considered transport hub.

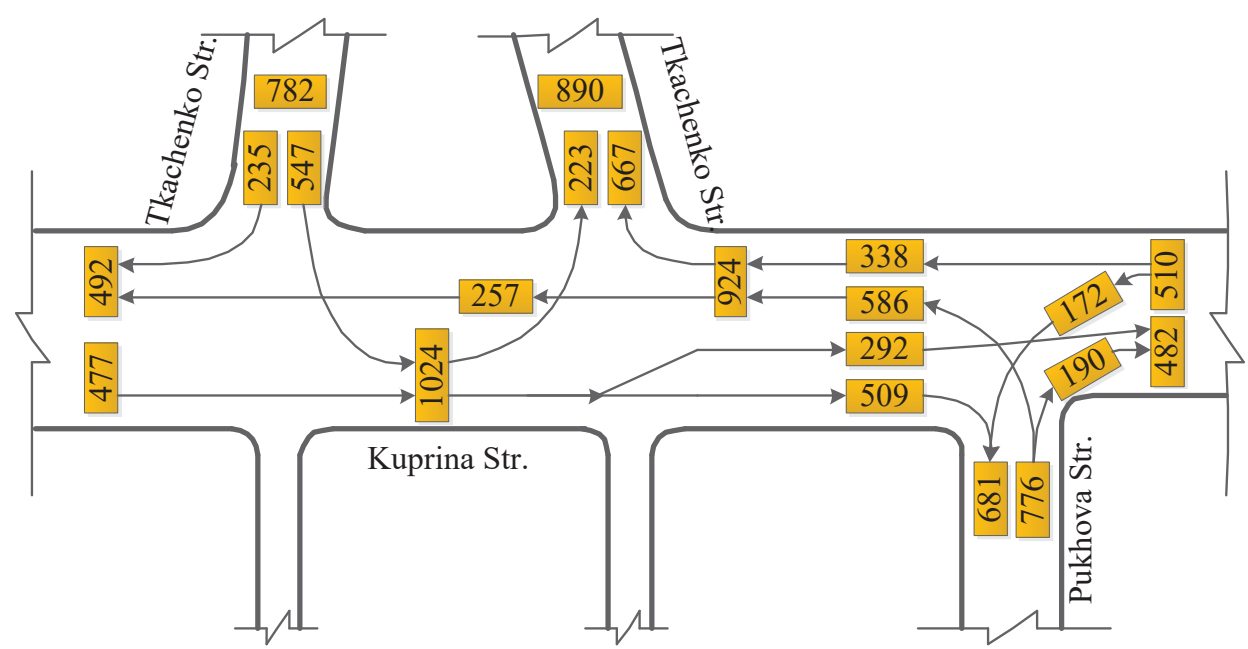

Fig. 2. The cyphergram of traffic flow intensities in the transport hub after the transformation 


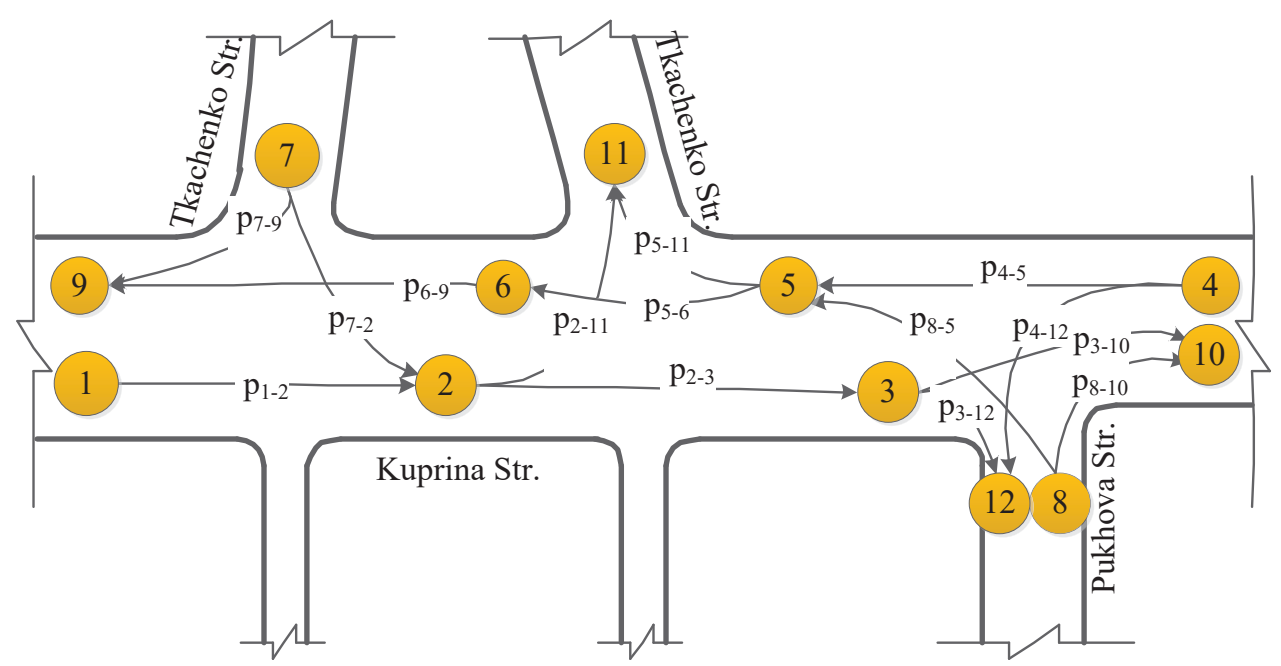

Fig. 3. The model of public transport movement in the transport hub with stops

On the graph, the state-based transition displays the movement of public transport with stops within the considered hub in certain directions (Table 1), and states 5, 10 and 14 denote public transport stops.

Table 1

Transition through the graph states for public transport routes

\begin{tabular}{|c|c|}
\hline $\begin{array}{c}\text { Transition through the } \\
\text { graph states }\end{array}$ & Vehicle route \\
\hline $1-2-3-4-5-6$ & $\begin{array}{c}\text { Trolleybus routes } 17 \text { and } 21 \\
\text { Bus route } 17\end{array}$ \\
\hline $7-8-9-10-11-12$ & $\begin{array}{c}\text { Trolleybus routes } 17 \text { and } 21 \\
\text { (reverse direction) } \\
\text { Bus route } 17 \text { (reverse direction) }\end{array}$ \\
\hline $12-14-15-2-3-16$ & Bus routes $37,50,79,80$, and $80 \mathrm{~A}$ \\
\hline $12-14-15-2-3-4-5-6$ & Bus route 53 \\
\hline
\end{tabular}

The matrix of transition probabilities for the reduced graph has the form

$$
\left\|p_{i j}^{*}\right\|=\left(\begin{array}{ccccccccc}
0 & 1 & 0 & 0 & 0 & 0 & 0 & 0 & 0 \\
0 & 0 & 0.742 & 0 & 0 & 0 & 0 & 0 & 0 \\
0 & 0 & 0 & 0 & 0 & 0 & 0 & 0 & 0 \\
0 & 0 & 0 & 0 & 0.625 & 0 & 0 & 0 & 0 \\
0 & 0 & 0 & 0 & 0 & 0.236 & 0 & 0 & 0 \\
0 & 0 & 0 & 0 & 0 & 0 & 0 & 0 & 1 \\
0 & 0.651 & 0 & 0 & 0 & 0 & 0 & 0 & 0.349 \\
0 & 0 & 0 & 0 & 0.755 & 0 & 0 & 0 & 0 \\
0 & 0 & 0 & 0 & 0 & 0 & 0 & 0 & 0 \\
0 & 0 & 0 & 0 & 0 & 0 & 0 & 0 & 0 \\
0 & 0 & 0 & 0 & 0 & 0 & 0 & 0 & 0 \\
0 & 0 & 0 & 0 & 0 & 0 & 0 & 0 & 0
\end{array}\right.
$$

The matrix of transition probabilities (1) was constructed for vehicles moving without stopping (i.e., for all vehicles except public transport) in the considered transport hub according to the model shown in Fig. 2.

\section{Construction of a simulation model for choosing the optimal operating conditions for vehicles for a section of the transport network}

To model the movement of vehicles, a safe distance model based on Gibbs' reflective model was used [26, 27]. In this model of following the leader, vehicles are of two types: without restriction and with restriction by the vehicle in front (the leader). If a vehicle has a leader, it will adapt its speed to maintain a safe distance from the leader. In other words, the safe distance is determined so that the vehicle can avoid a collision with any adequate actions of its leader. If there is no restriction for the vehicle, then its speed is limited by the desired velocity and the maximum possible acceleration.

The speed of the $n$-th vehicle in the time interval $[t, t+T]$ is determined as follows

$$
v_{n}(t+T)=\min \left\{v_{n}^{a}(t+T), v_{n}^{b}(t+T)\right\},
$$

where $T$ is the driver's reaction time, which is equal to one step of time simulation.

$$
\left.\begin{array}{ccc}
0 & 0 & 0 \\
0 & 0.258 & 0 \\
0.293 & 0 & 0.707 \\
0 & 0 & 0.375 \\
0 & 0.764 & 0 \\
0 & 0 & 0 \\
0 & 0 & 0 \\
0.245 & 0 & 0 \\
0 & 0 & 0 \\
0 & 0 & 0 \\
0 & 0 & 0 \\
0 & 0 & 0
\end{array}\right)
$$

The maximum velocity $v_{n}^{a}$, that a vehicle can develop in a time step is determined by the following expression:

(1)

$$
\begin{aligned}
& v_{n}^{a}(t+T)=v_{n}(t)+ \\
& +2.5 \cdot a_{n}^{\max } \cdot T \times \\
& \times\left(1-\frac{v_{n}(t)}{v_{n}^{\text {des }}}\right) \times \\
& \times \sqrt{0.025+\frac{v_{n}(t)}{v_{n}^{d e s}},}
\end{aligned}
$$

where $a_{n}^{\max }$ is the maximum value of acceleration of the $n$-th vehicle during its acceleration, and $v_{n}^{\text {des }}$ is the desired velocity of the $n$-th vehicle.

The safest velocity $v_{n}^{b}$ of the $n$-th vehicle, taking into account the leading vehicle, is determined by the formula 


$$
\begin{aligned}
& v_{n}^{b}(t+T)=d_{n}^{\max } \cdot T+ \\
& +\sqrt{\left(d_{n}^{\max } \cdot T\right)^{2}-d_{n}^{\max } \cdot\left\{\begin{array}{l}
2\left[x_{n-1}(t)-s_{n-1}-x_{n}(t)\right]- \\
-v_{n}(t) \cdot T-\frac{v_{n-1}(t)^{2}}{\hat{d}_{n-1}}
\end{array}\right\}},
\end{aligned}
$$

where $d_{n}^{\max }$ is the maximum value of acceleration of the $n$-th vehicle during its braking, $x_{n}$ is the coordinate of the $n$-th vehicle, $s_{n-1}$ is the effective length of the $(n-1)$-th vehicle, which is determined by the length of the vehicle and the parameter that sets the minimum distance between vehicles, and $\hat{d}_{n-1}$ is the estimate of the maximum acceleration of the $(n-1)$-th vehicle during its braking.

There are several approaches to assessing the acceleration of the leader when it is braking. First, it can be assumed that the driver can accurately estimate the leader's deceleration, thus assuming that

$$
\hat{d}_{n-1}=d_{n-1},
$$

where $d_{n-1}$ is the acceleration of the $(n-1)$-th vehicle during braking. Second, the deceleration estimate of the lead vehicle can be calculated as the average between the acceleration of the lead and follower vehicles:

$$
\hat{d}_{n-1}=\frac{d_{n-1}+d_{n}}{2} .
$$

An algorithm is thus suggested for the development and verification of a simulation model for optimizing the operating conditions of vehicles in the transport hub, which is shown in Fig. 4.

At the next stage of developing the simulation model, a road network of the researched transport hub was created on the basis of a real scheme, and a process diagram of the simulation model was constructed according to [23].

Next, the process diagram was developed for the simulation model of the movement of public transport vehicles in the researched hub, running with stops according to the previously developed model shown in Fig. 3.

Thus, Fig. 5 shows the process diagram for public transport going in the following directions (Fig. 3): $12-14-15-2-3-4-5-6$ to simulate the movement of route taxis on route $53 ; 1-2-3-4-5-6$ to simulate the movement of trolleybuses on routes 17 and 21, as well as route taxis on route 17.

Fig. 6 shows the process diagram for public transport going in the direction $7-8-9-10-11-12$ to simulate the reverse direction of trolleybuses on routes 17 and 21, as well as route taxis on route 17 .
Fig. 7 shows the process diagram for public transport going in the direction $12-14-15-2-3-16$ when simulating the traffic of route taxis on routes $37,50,79,80$, and $80 \mathrm{~A}$ and buses on route 80 .

The resulting process diagram of the simulation model to optimize the traffic in the transport hub is shown in Fig. 8.

Fig. 4. The flowchart of the algorithm of the simulation model for choosing the optimal operating conditions for vehicles in the transport hub

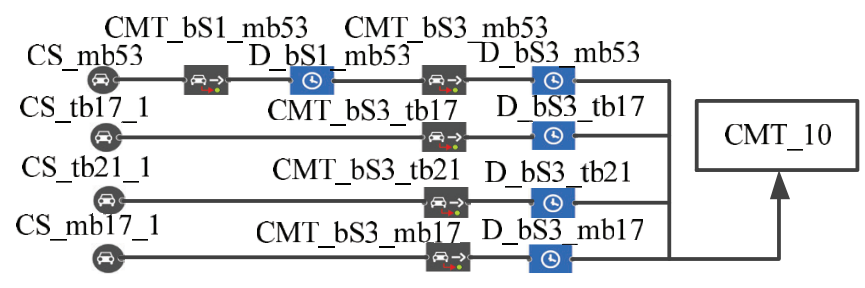

Fig. 5. The process diagram of the simulation model for public transport running on routes $12-14-15-2-3-4-5-6$ and

$$
1-2-3-4-5-6
$$

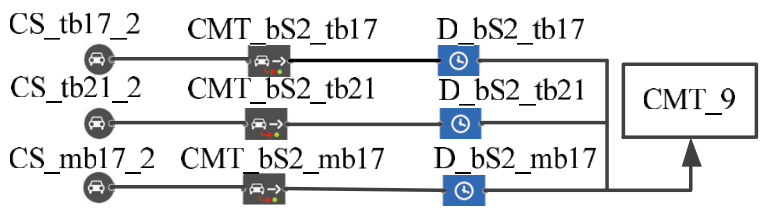

Fig. 6 . The process diagram of the simulation model for public transport running on routes $7-8-9-10-11-12$ 


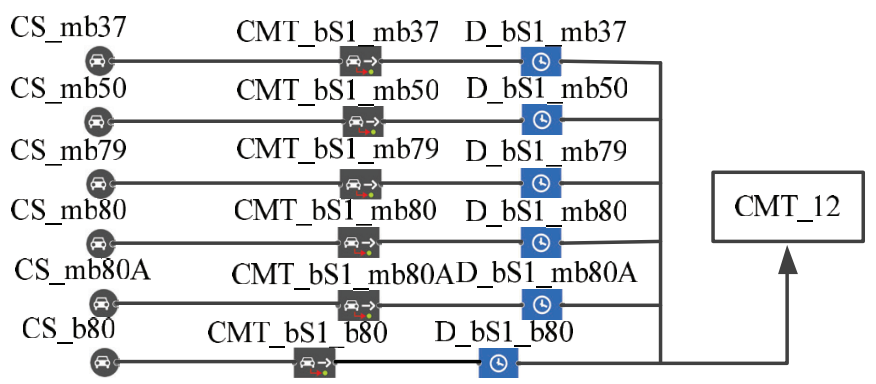

Fig. 7. The process diagram of the simulation model for public transport running on routes $12-14-15-2-3-16$

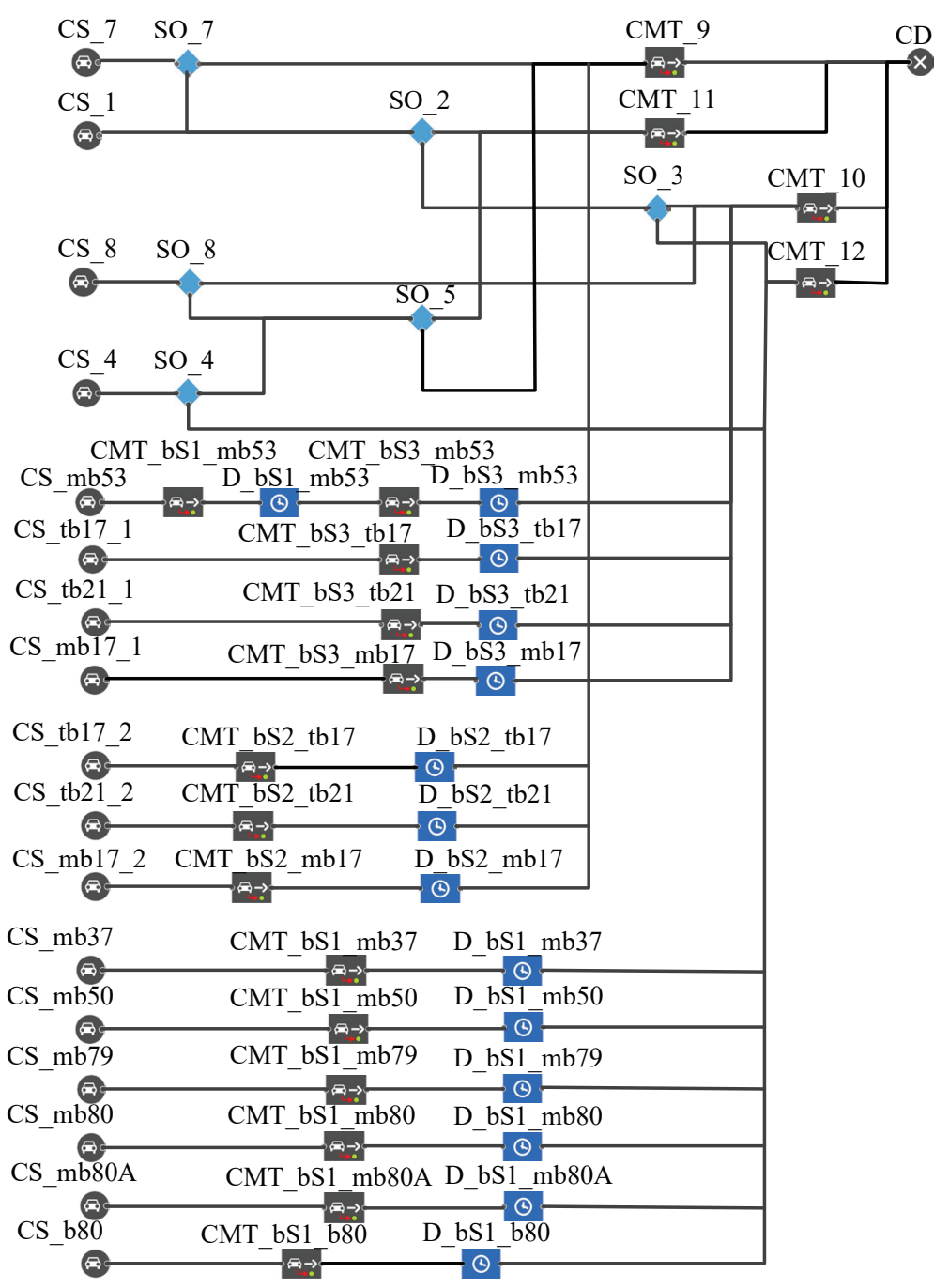

Fig. 8. The process diagram of the simulation model to optimize the traffic in the transport hub

In the process of constructing the process diagrams of the simulation model, the following blocks were used:

- CS_1, CS_4, CS_7, and CS_8 are the incoming streams for the model of the considered transport hub for the graph points $1,4,7$, and 8 , respectively;

- $\mathrm{SO} \_2, \mathrm{SO} \_3, \mathrm{SO} \_4, \mathrm{SO}$ 5, SO_7, and $\mathrm{SO} \_8$ are the blocks intended for dividing the flows entering them into two streams for the model of the considered transport hub for the graph points $2,3,4,5,7$, and 8 , respectively;

-CMT_9, CMT_10, CMT_11, and CMT_12 are the blocks displaying outgoing flows for the model of the con- sidered transport hub for the graph points 9, 10, 11, and 12 , respectively;

- CS mb53, CS mb17 1, CS mb17 2, CS mb37, CS mb50, CS mb79, CS mb80, and CS mb80A are the incoming flows for the model according to Fig. 3 for point 13 of the graph for route taxis on routes 53, 17, 17 (reverse direction), 37, 50, 79, 80, and 80A, respectively;

- CS_tb17_1, CS_tb21_1, CS_tb17_2, and CS tb21_2 are the incoming flows for the model according to Fig. 3 for points 1 and 7 of the graph for trolleybuses on routes 17, 21, 17 (reverse direction), and 21 (reverse direction), respectively;

- CS_b80 shows the incoming flows for the model according to Fig. 3 for point 13 of the graph for the bus on route 80 ;

-CMT_bS1_mb53, CMT_bS1_mb37, CMT_bS1_mb50, CMT_bS1_mb79, CMT bS1_mb80, CMT_bS1_mb80A, and CMT_ bS1_b80 are the blocks leading to a stop corresponding to point 14 of the graph according to the model shown in Fig. 3 for such vehicles: route taxis on routes 53,37 , $50,79,80$, and $80 \mathrm{~A}$ and buses on route 80 , respectively;

- CMT bS2 tb17, CMT bS2 tb21, and CMT bS2 2 mb17 are the blocks leading to the stop corresponding to point 5 of the graph according to the model shown in Fig. 3 for such vehicles: trolleybuses on routes 17 and 21 and route taxi on route 17 , respectively;

- CMT bS3 mb53, CMT bS3 mb17, CMT_bS3 tb17, and CMT_bS3 tb21 are the blocks leading to a stop corresponding to point 10 of the graph according to the model shown in Fig. 3 for such vehicles: route taxis on routes 53 and 17 and trolleybuses on route 17 and 21, respectively;

- D_bS1_mb53, D_bS1_mb37, DTo bS1_mb50, D_bS1_mb7 $\overline{9}, \mathrm{D} \overline{\mathrm{b}} \mathrm{D} 1 \mathrm{mb} 80, \mathrm{D}$ bS1_mb80A, and D_bS1_b80 are the blocks that set the delay time at the stop corresponding to point 14 of the graph according to the model shown in Fig. 3 for such vehicles: route taxis on route $53,37,50,79,80$, and $80 \mathrm{~A}$ and buses on route 80 , respectively;

- D_bS2_tb17, D_bS2_tb21, and D_ bS2_mb17 are the blocks that set the delay time at the stop corresponding to point 5 of the graph according to the model shown in Fig. 3 for such vehicles: trolleybuses on routes 17 and 21 and route taxis on route 17, respectively;

- D_bS3_mb53, D_bS3_mb17, D_bS3 tb17, and D bS3 tb21 are the blocks that set the delay time at the stop corresponding to point 10 of the graph according to the model shown in Fig. 3 for such vehicles: route taxis on route 53 and 17 and trolley buses on routes 17 and 21, respectively;

- CD is the block that removes vehicles from the system.

While implementing the model, new types of agents were created for a vehicle (Car), a route taxi (MBus), a bus (Bus) and a trolleybus (TBus) with parameters reflecting the time spent by each agent in the system, $t \_b s \_c, t \_s \_m b, t b s \_b$, $t \_t b$, respectively, and also the corresponding populations 
of the agents (cars, mBuses, Buses, and tBuses) that were added to blocks of the carSource (CS) type.

To collect data on the travel time of the agents of the transport hub, the travel time element was created, which estimates the difference between the current time () and the time when the agents appear in the system, that is,

$$
\begin{aligned}
& \text { travel_time }= \\
& =\left\{\begin{array}{l}
\text { main.travel_time.add }\left(\text { time }()-t_{-} b s_{-}\right) \\
\text {main.travel_time.add }\left(\text { time }()-t_{-} b s_{-} m b\right) \\
\text { main.travel_time.add }\left(\text { time }()-t_{-} b s_{-} b\right) \\
\text { main.travel_time.add }\left(\text { time }()-t_{-} b s_{-} t b\right)
\end{array}\right\} .
\end{aligned}
$$

The duration of the traffic light phases was set by the parameters $p 1$ and $p 2$ with default values of 37 and 25 , respectively.

To display traffic light regulation, four Traffic Light blocks were added to the system. In the Properties of these blocks, the operation modes of the traffic light objects for the specified stop lines were set. The blocks were divided into groups of directions of movement (Fig. 9). The modes of operation of the traffic light objects were expressed in general terms through the parameters of the duration of the phases of the traffic light objects $p 1$ and $p 2$, taking into account the transient modes for the possibility of further optimization of road traffic in the hub under consideration.

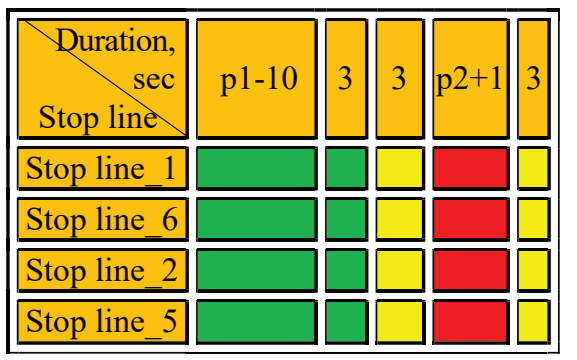

a

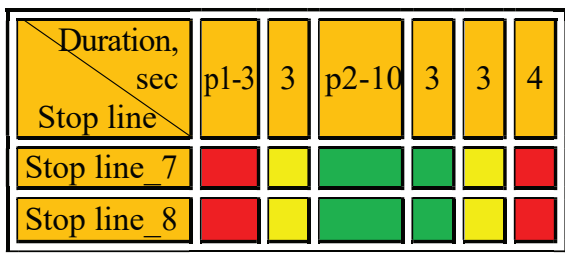

b
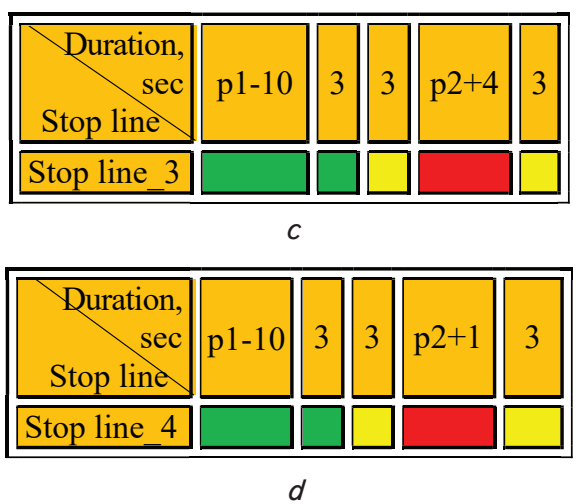

Fig. 9. Operation modes of traffic light objects for specified stop lines: $a-$ trafficLight_1_2_5_6; $b-$ trafficLight_7_8; $c$ - trafficLight_3; $d-$ trafficLight_4
To optimize traffic in the transport hub, an experiment 'Optimization' was created based on the model in Fig. 1. The optimization problem was reduced to minimizing the objective function travel_time $(p 1, p 2)$, that is,

$$
\begin{aligned}
& \text { travel_time }(p 1, p 2)=\sum_{i} \sum_{j} \Delta t_{i j}= \\
& =\sum_{j}^{k_{1}}\left[t{ }_{-} b s_{-} c_{j}(p 1, p 2)-t o_{1 j}\right]+ \\
& +\sum_{j}^{k_{2}}\left[t t_{-} b s_{-} m b_{j}(p 1, p 2)-t o_{2 j}\right]+ \\
& +\sum_{j}^{k_{3}}\left[t t_{-} b s_{-} b_{j}(p 1, p 2)-t o_{3 j}\right]+ \\
& +\sum_{j}^{k_{4}}\left[t t_{-} b s_{-} t b_{j}(p 1, p 2)-t o_{4 j}\right] \rightarrow \min ,
\end{aligned}
$$

where $i$ is the parameter characterizing the type of agent in the simulation model, $j$ is the parameter characterizing the number of the $i$-th agent in the population of the model agents, and $k_{i}$ is the number of $i$-th agents in the population of the agents.

$$
i=\left(\begin{array}{c}
\text { Car } \\
M B u s \\
B u s \\
\text { TBus }
\end{array}\right) .
$$

The restrictions imposed on the model are reduced to the system:

$$
\left\{\begin{array}{l}
1 \leq i \leq n, \\
0 \leq j \leq k_{i}, \\
p 1_{\min } \leq j \leq p 1_{\max }, \\
p 2_{\min } \leq j \leq p 2_{\max }, \\
\sum_{i} k_{i}=k o,
\end{array}\right.
$$

where $p 1_{\min }, p 2_{\min }, p 1_{\max }$, and $p 2_{\max }$ are, respectively, the minimum and maximum values of the parameters $p 1$ and $p 2$, and $k o$ is the total number of agents in the model.

Optimization of the model consists in the sequential performance of several runs of the model with different values of the parameters and finding the optimal values of the parameters for the given task. It is necessary to estimate the best values of the model parameters taking into account the given constraints. By combining heuristics, neural networks and mathematical optimization, the values of the model parameters corresponding to the maximum or minimum of the objective function were found, both under uncertainty and under constraints.

The interface of the optimization window of the simulation model was created, in which its number, functionality and parameters $p 1$ and $p 2$ were displayed in the form of a table for the current and best values of the iteration.

\section{Results of modelling and optimizing vehicle operating conditions for the researched section of the transport network}

In the optimization process, a triangular distribution was used to model public transport delays at stops. A tri- 
angular distribution is a continuous distribution bounded on both sides. It is often used when there is little or no information available and can rarely accurately represent a set of values. Despite this, due to its ease of use, it is applied as a functional form of representing areas with fuzzy logic.

The function of the distribution density of the triangular distribution (Fig. 10, $a$ ) is

$$
\begin{aligned}
& f(x)= \\
& = \begin{cases}0 & \text { for } x<x_{\min } \\
\frac{2\left(x-x_{\min }\right)}{\left(x_{\max }-x_{\min }\right)\left(x_{\bmod }-x_{\min }\right)} & \text { for } x_{\min } \leq x \leq x_{\bmod }, \\
\frac{2\left(x_{\max }-x\right)}{\left(x_{\max }-x_{\min }\right)\left(x_{\max }-x_{\bmod }\right)} & \text { for } x_{\bmod }<x \leq x_{\max }, \\
0 & \text { for } x>x_{\max } .\end{cases}
\end{aligned}
$$

The distribution function of the triangular distribution (Fig. 10,b) is

$$
\begin{aligned}
& F(x)= \\
& = \begin{cases}0 & \text { for } x<x_{\min }, \\
\frac{\left(x-x_{\min }\right)^{2}}{\left(x_{\max }-x_{\min }\right)\left(x_{\bmod }-x_{\min }\right)} & \text { for } x_{\min } \leq x \leq x_{\bmod }, \\
1-\frac{\left(x_{\max }-x\right)^{2}}{\left(x_{\max }-x_{\min }\right)\left(x_{\max }-x_{\bmod }\right)} & \text { for } x_{\bmod }<x \leq x_{\max }, \\
1 & \text { for } x>x_{\max } .\end{cases}
\end{aligned}
$$

The triangular distribution was specified by the triangular (double min, double mode, and double max) function, which generated the $x$ value according to the triangular distribution (Table 2). For the delay (D) blocks, the corresponding parameters of the triangular distributions are given in Table 3.

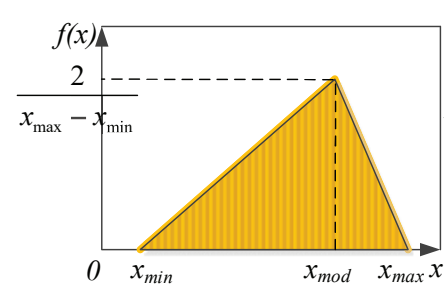

a

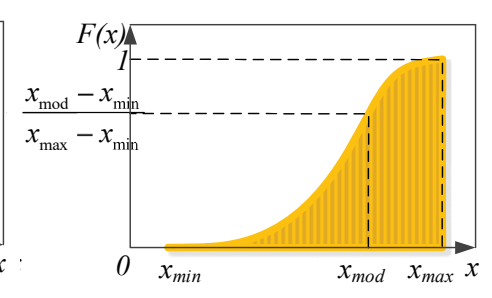

b
Fig. 10. The function of the triangular distribution: $a-$ the function of the distribution density; $b$ - the distribution function

Table 2

Description of the parameters and result of the triangular (double min, double mode, and double max) function

\begin{tabular}{|c|c|c|}
\hline Name & Type & Description \\
\hline \multicolumn{2}{|c|}{ Parameters } \\
\hline min & Double & the minimum value of $x$ \\
\hline $\max$ & Double & the maximum value of $x$ \\
\hline mode & Double & the most likely value of $x$ \\
\hline \multicolumn{2}{|c|}{ Result } \\
\hline$X$ & Double & generated value \\
\hline
\end{tabular}

Table 3

Parameters of the triangular distributions for the delay blocks $(D)$

\begin{tabular}{|c|c|}
\hline Block $D$ & Delay time distribution function \\
\hline D_bS1_mb53 & triangular $(0,8,20)$ \\
\hline D_bS3_mb53 & triangular $(5,8,15)$ \\
\hline D_bS3_tb17 & triangular $(7,12,20)$ \\
\hline D_bS3_tb21 & triangular $(5,10,19)$ \\
\hline D_bS3_mb17 & triangular $(0,9,12)$ \\
\hline D_bS2_tb17 & triangular $(7,13,15)$ \\
\hline D_bS2_tb21 & triangular $(6,13,16)$ \\
\hline D_bS2_mb17 & triangular $(5,8,15)$ \\
\hline D_bS1_mb37 & triangular $(5,7,14)$ \\
\hline D_bS1_mb50 & triangular $(7,9,14)$ \\
\hline D_bS1_mb79 & triangular $(0,7,10)$ \\
\hline D_bS1_mb80 & triangular $(6,9,11)$ \\
\hline D_bS1_mb80A & triangular $(0,6,10)$ \\
\hline D_bS1_b80 & triangular $(5,12,16)$ \\
\hline
\end{tabular}

During the optimization experiment, the parameters $p 1$ and $p 2$ were varied in the range from $20 \mathrm{~s}$ to $40 \mathrm{~s}$ with a step of $1 \mathrm{~s}$.

Table 4 and Fig. $11 a$ show the results of the simple experiment 'Simulation1', which simulated traffic in the considered transport hub with the current values of $p 1=37 \mathrm{~s}$ and $p 2=25 \mathrm{~s}$ with the given vehicles according to the model in [23].

Table 4 and Figs. 11, $b, 12, a$ show the results of the simple experiment 'Simulation2', which simulated traffic in the considered transport hub with the current values of $p 1=37 \mathrm{~s}$ and $p 2=25 \mathrm{~s}$. However, it takes into account public transport stops according to the model in Fig. 3 and the matrix of the transition probabilities $p_{i j}^{*}$ (1).

The results of traffic optimization in the considered transport hub are shown in Fig. 13.

It was found that for a given objective function (7), the optimal phase values were $p 1=37 \mathrm{~s}$ and $p 2=20 \mathrm{~s}$, which were obtained for the sixth iteration. In this case, the average travel time of a vehicle through the hub under consideration was about $117 \mathrm{~s}$, and the average relative error of real tests with the simulation modelling results was $3.2 \%$. The maximum error value was $4.3 \%$.

Table 4

Results of simulating travel_time

\begin{tabular}{|c|c|c|c|}
\hline Parameter & $\begin{array}{c}\text { Duration } \\
\text { for "Simula- } \\
\text { tion1" }\end{array}$ & $\begin{array}{c}\text { Duration } \\
\text { for "Simula- } \\
\text { tion2" }\end{array}$ & $\begin{array}{c}\text { Duration } \\
\text { for "Simula- } \\
\text { tion3" }\end{array}$ \\
\hline $\begin{array}{c}\text { Number of vehicles } \\
\text { passing through } \\
\text { the hub }\end{array}$ & 1,160 & 1,147 & 1,096 \\
\hline $\begin{array}{c}\text { Average trav- } \\
\text { el_time, s }\end{array}$ & 136.846 & 131.283 & 117.088 \\
\hline $\begin{array}{c}\text { Minimum travel_- } \\
\text { time, s }\end{array}$ & 15.766 & 15.66 & 15.66 \\
\hline $\begin{array}{c}\text { Maximum travel_ } \\
\text { time, } s\end{array}$ & $1,182.576$ & 646.449 & 612.859 \\
\hline $\begin{array}{c}\text { Standard deviation } \\
\text { of travel time, } \mathrm{s}\end{array}$ & 148.766 & 106.978 & 105.796 \\
\hline $\begin{array}{c}\text { Confidence interval } \\
\text { of travel time, } \mathrm{s}\end{array}$ & 8.561 & 6.191 & 6.264 \\
\hline Total travel_time, $\mathrm{s}$ & $158,741.588$ & $150,581.158$ & $128,326.621$ \\
\hline
\end{tabular}




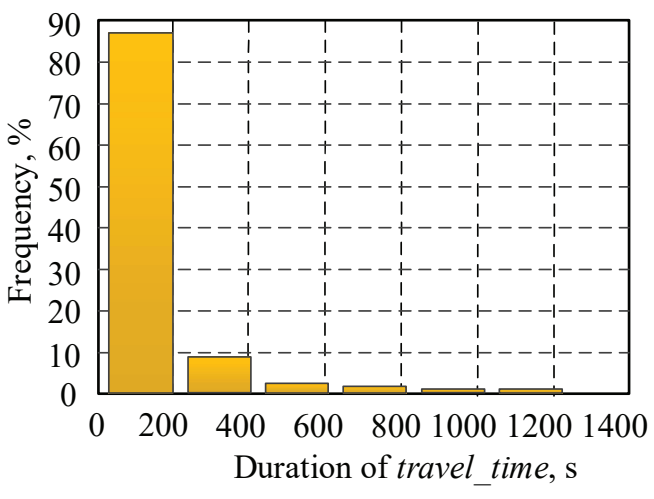

$a$

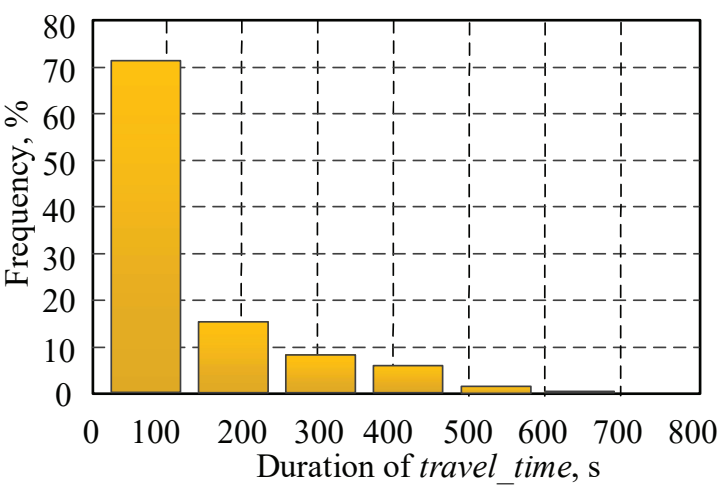

$b$

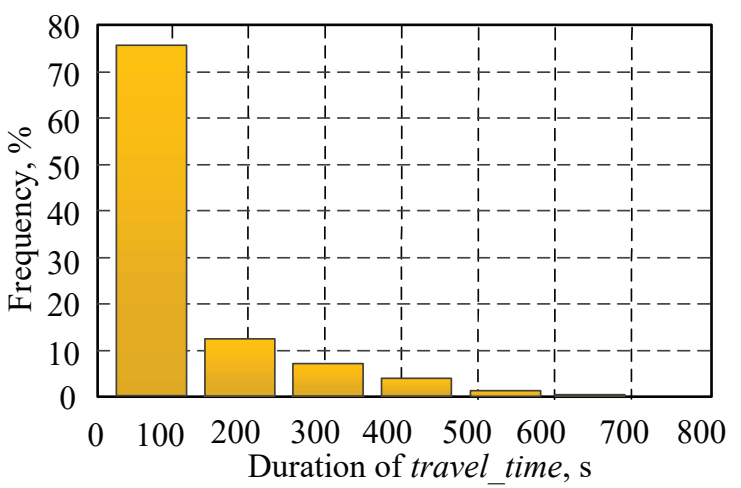

$c$

Fig. 11. The histogram of the probable trave/_time distribution for the experiments: $a$ - 'Simulation1'; $b$ - 'Simulation2'; $c-$ 'Simulation3'

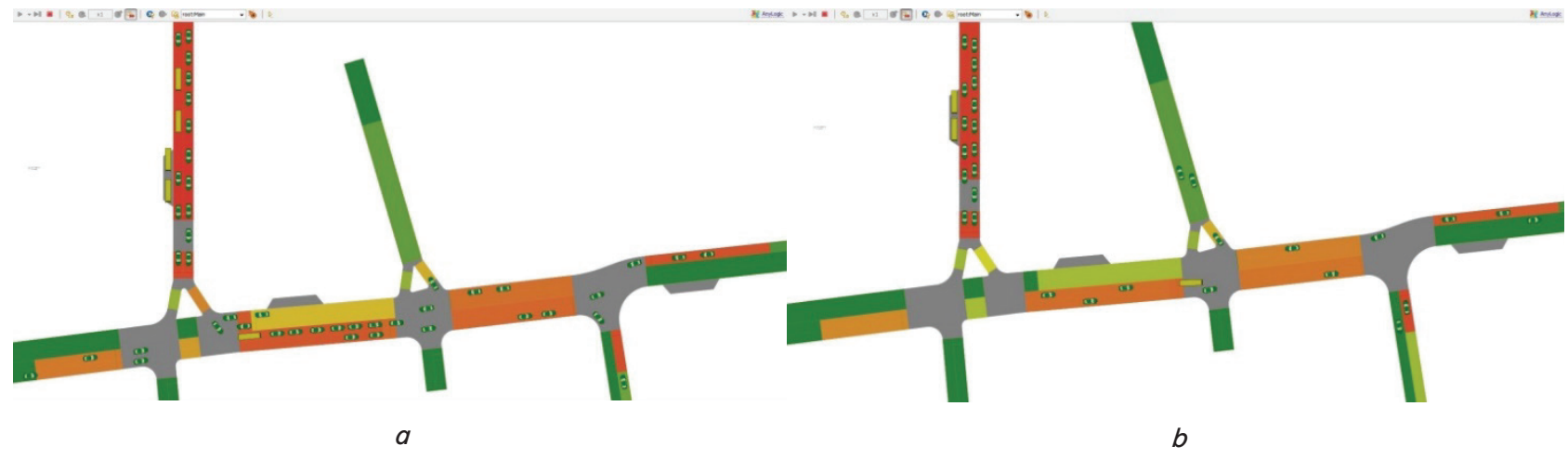

Fig. 12. The map of traffic jams in the simulation model for the experiments: $a$ - 'Simulation2'; $b$ - 'Simulation3'

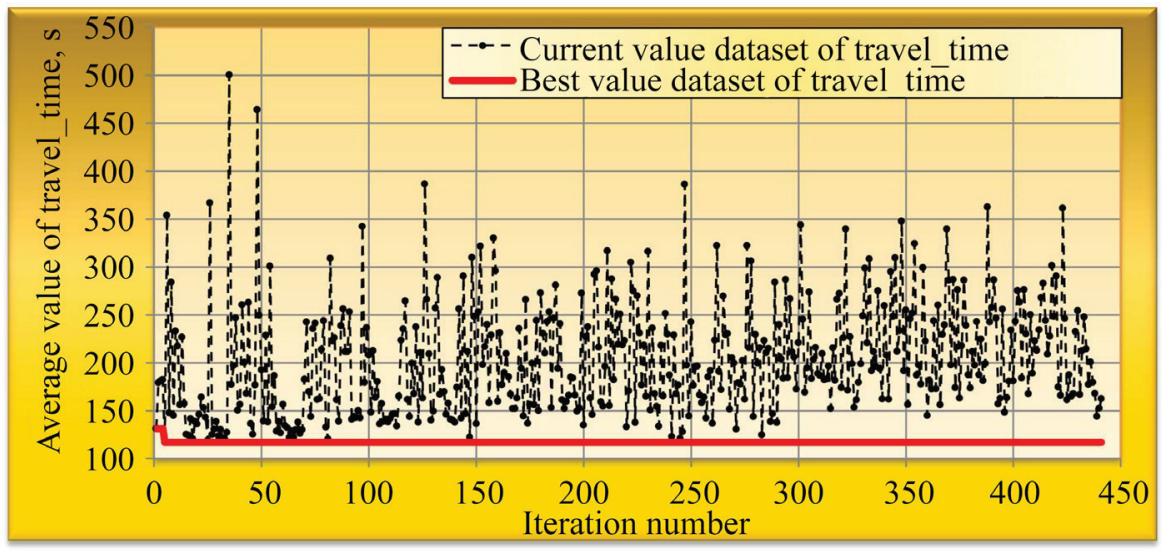

Fig. 13. The results of traffic optimization in the transport hub 
Table 4 and Figs. 11, $c, 12, b$ show the results of the simple experiment 'Simulation3', which simulated road traffic in the considered transport hub with the optimal values of $p 1=37 \mathrm{~s}$ and $p 2=20 \mathrm{~s}$ and taking into account public transport stops according to the model in Fig. 3 and the matrix of transition probabilities $p_{i j}^{*}(1)$.

Fig. 11-13 show that the selection of public transport vehicles from the total number, taking into account their stops at stopping points, affects the modelling of traffic in the hub.

\section{Discussion of the results of modelling and optimizing the vehicle operating conditions}

Based on the comparison of the average relative error of real tests with the simulation modelling results, it can be concluded that the simulation model adequately describes real processes. At the same time, the maximum error value of $4.3 \%$ is due to the fact that, as a result of simulation modelling, the selected vehicle movement model slightly differs from the real one. This is due, inter alia, to the culture of operating the vehicles. As a measured parameter, we took the average travel time of the vehicles in the transport hub. The measurements were performed at the same points at which the vehicles entered and left the transport hub according to the simulation model.

Thus, as the optimization result, the average travel time through the considered transport hub was reduced by $10.8 \%$ (Table 4), and the number of vehicles in traffic jams was reduced by $11.5 \%$ (Fig. 12), which shows the effectiveness of the developed models.

The developed simulation models can be used in the process of rebuilding a transport hub to simulate traffic under changing operation conditions for its vehicles and to predict them. They can also be effective in the process of managing the operating conditions of vehicles in the information analysis system V2I of monitoring and control proposed by the authors.

The implementation of the described method for managing vehicles with changes in the atmospheric and climatic conditions of operation and the culture of operation requires further research.

The study took into account changes in transport and road conditions for the operation of vehicles. The influence of public transport on the results of the simulation modelling presented in the work was also researched. At the same time, the atmospheric and climatic conditions of operation and the culture of operation of vehicles were taken constant, although they had been included in the model. In the future, it is planned to take into account the influence of these factors in dynamics.

\section{Conclusions}

1. A dynamic model has been built for determining the optimal parameters of the operation of vehicles in a transport hub, which takes into account a large number of factors affecting the process of movement. The model can be used in the formation of an intelligent transport system of a city to integrate the software in the infrastructure of the transport network.

2. The operating conditions for vehicles in the researched transport hub were analysed. Graphic models of the movement of the vehicles in it were built and the influence of public transport on the process of movement in the hub was considered. The obtained models and the values of the vehicle operation parameters were taken into account when performing the simulation modelling.

3. A simulation model has been created for choosing the optimal operating conditions for vehicles, taking into account the peculiarities of public transport, which can be used to solve the problems of optimizing the movement of vehicles. The model was tested on a section of a transport network. Its adequacy was confirmed by appropriate calculations. As a result of the optimization, the average travel time through the considered transport hub and the number of vehicles in traffic congestion were reduced. The developed simulation models can be used in the process of rebuilding a transport hub to simulate traffic under changing operation conditions for its vehicles and to predict them.

\section{References}

1. Gritsuk, I. V., Volkov, V., Mateichyk, V., Grytsuk, Y., Nikitchenko, Y., Klets, D. et. al. (2018). Information Model of V2I System of the Vehicle Technical Condition Remote Monitoring and Control in Operation Conditions. SAE Technical Paper Series. doi: https://doi.org/10.4271/2018-01-0024

2. Quan, J., Zhao, Y., Tan, G., Xu, Y., Huang, B., He, T. (2018). A Study on Safety Intelligent Driving System for Heavy Truck Downhill in Mountainous Area. SAE Technical Paper Series. doi: https://doi.org/10.4271/2018-01-1887

3. Senapati, P. R. R., Das, S., Vora, P. B. (2017). Intelligent Braking and Maneuvering System for an Automobile Application. SAE Technical Paper Series. doi: https://doi.org/10.4271/2017-26-0080

4. Gritsuk, I. V., Mateichyk, V., Aleksandrov, V., Prilepsky, Y., Panchenko, S., Kagramanian, A. et. al. (2019). Features of Modeling Thermal Development Processes of the Vehicle Engine Based on Phase-Transitional Thermal Accumulators. SAE Technical Paper Series. doi: https://doi.org/10.4271/2019-01-0906

5. Gritsuk, I., Pohorletskyi, D., Mateichyk, V., Symonenko, R., Tsiuman, M., Volodarets, M. et. al. (2020). Improving the Processes of Thermal Preparation of an Automobile Engine with Petrol and Gas Supply Systems (Vehicle Engine with Petrol and LPG Supplying Systems). SAE Technical Paper Series. doi: https://doi.org/10.4271/2020-01-2031

6. Golovan, A., Gritsuk, I., Popeliuk, V., Sherstyuk, O., Honcharuk, I., Symonenko, R. et. al. (2019). Features of Mathematical Modeling in the Problems of Determining the Power of a Turbocharged Engine According to the Characteristics of the Turbocharger. SAE International Journal of Engines, 13 (1). doi: https://doi.org/10.4271/03-13-01-0001

7. Osipkov, V., Ksenevich, T. I., Belousov, B., Karasev, O., Sokolov, S., Rudynets, M. et. al. (2016). Intelligent Transport Systems: Revolutionary Threats and Evolutionary Solutions. SAE Technical Paper Series. doi: https://doi.org/10.4271/2016-01-0157 
8. Kiselev, A. B., Kokoreva, A. V., Nikitin, V. F., Smirnov, N. N. (2004). Mathematical modelling of traffic flows on controlled roads. Journal of Applied Mathematics and Mechanics, 68 (6), 933-939. doi: https://doi.org/10.1016/j.jappmathmech.2004.11.014

9. Kelarestaghi, K. B., Heaslip, K., Khalilikhah, M., Fuentes, A., Fessmann, V. (2018). Intelligent Transportation System Security: Hacked Message Signs. SAE International Journal of Transportation Cybersecurity and Privacy, 1 (2), 75-90. doi: https://doi.org/ 10.4271/11-01-02-0004

10. Županović, D., Anžek,M., Kos, G.(2012). Optimisation of Signal-controlled Intersection Capacity.PROMET - Traffic\&Transportation, 22 (6), 419-431. doi: https://doi.org/10.7307/ptt.v22i6.207

11. Zhao, F., Tan, H., Liu, Z. (2020). Safety Development Trend of the Intelligent and Connected Vehicle. SAE Technical Paper Series. doi: https://doi.org/10.4271/2020-01-0085

12. Xu, Z. (2017). Macroscopic Traffic States Estimation Based on Vehicle-to-Infrastructure (V2I) Connected Vehicle Data. SAE Technical Paper Series. doi: https://doi.org/10.4271/2017-01-2013

13. Guseynov, S. E., Berezhnoy, A. V. (2017). Modelling of urban traffic flow. Environment. Technology. Resources. Proceedings of the International Scientific and Practical Conference, 1, 109. doi: https://doi.org/10.17770/etr2017vol1.2632

14. Li, J., Wu, J., Sun, H., Jiang, Y., Deng, W., Zhu, B. (2017). Traffic Modeling Considering Motion Uncertainties. SAE Technical Paper Series. doi: https://doi.org/10.4271/2017-01-2000

15. Kim, N., Karbowski, D., Rousseau, A. (2018). A Modeling Framework for Connectivity and Automation Co-simulation. SAE Technical Paper Series. doi: https://doi.org/10.4271/2018-01-0607

16. Kerner, B. S. (2009). Introduction to Modern Traffic Flow Theory and Control: The Long Road to Thre. Springer. doi: https://doi.org/ 10.1007/978-3-642-02605-8

17. Dhanavanthan, B. (2017). Propagation Loss Measurements and Two Slope Modelling in Vehicular Environments for Intelligent Transportation Systems. SAE Technical Paper Series. doi: https://doi.org/10.4271/2017-01-0093

18. Hull, T. (2017). Intelligent Robotics Safeguarding. SAE International Journal of Engines, 10 (2), 215-221. doi: https://doi.org/ 10.4271/2017-01-0293

19. Wang, Y., Song, R., Yang, C. (2018). Research on Intelligent Vehicle Index and Evaluation Method. SAE Technical Paper Series. doi: https://doi.org/10.4271/2018-01-1634

20. Falendysh, A., Kharlamov, P., Kletska, O., Volodarets, N. (2016). Calculation of the Parameters of Hybrid Shunting Locomotive. Transportation Research Procedia, 14, 665-671. doi: https://doi.org/10.1016/j.trpro.2016.05.325

21. Falendysh, A., Volodarets, M., Kletska, O., Hatchenko, V. (2017). The impact of the type of operation on the parameters of a shunting diesel locomotive with hybrid power plant. MATEC Web of Conferences, 133, 03003. doi: https://doi.org/10.1051/ matecconf/201713303003

22. Gorobchenko, O., Fomin, O., Gritsuk, I., Saravas, V., Grytsuk, Y., Bulgakov, M. et. al. (2018). Intelligent Locomotive Decision Support System Structure Development and Operation Quality Assessment. 2018 IEEE 3rd International Conference on Intelligent Energy and Power Systems (IEPS). doi: https://doi.org/10.1109/ieps.2018.8559487

23. Volodarets, M., Gritsuk, I., Chygyryk, N., Belousov, E., Golovan, A., Volska, O. et. al. (2019). Optimization of Vehicle Operating Conditions by Using Simulation Modeling Software. SAE Technical Paper Series. doi: https://doi.org/10.4271/2019-01-0099

24. Gao, X., Deng, W., Wang, J. (2018). GPS Modeling for Vehicle Intelligent Driving Simulation. SAE International Journal of Connected and Automated Vehicles, 2 (1), 57-65. doi: https://doi.org/10.4271/2018-01-0763

25. Lin, Z., Guo, X., Pei, X., Yang, B., Zhang, Y. (2017). Dynamic Modeling and State Estimation for Multi-In-Wheel-Motor-Driven Intelligent Vehicle. SAE Technical Paper Series. doi: https://doi.org/10.4271/2017-01-1996

26. Kanchwala, H., Ogai, H. (2016). Development of an Intelligent Transport System for EV. SAE International Journal of Passenger Cars - Electronic and Electrical Systems, 9 (1), 9-21. doi: https://doi.org/10.4271/2015-01-9132

27. Khastgir, S., Dhadyalla, G., Birrell, S., Redmond, S., Addinall, R., Jennings, P. (2017). Test Scenario Generation for Driving Simulators Using Constrained Randomization Technique. SAE Technical Paper Series. doi: https://doi.org/10.4271/2017-01-1672 